\title{
Advances in Electron Holography for High-Resolution Electromagnetic Field Study
}

\section{Toshiaki Tanigaki and Hiroyuki Shinada}

Research \& Development Group, Hitachi, Ltd., Hatoyama, Japan

Observing structures and electromagnetic fields at atomic resolutions provides critical information for material science and industrial research. A transmission electron microscope (TEM) has advantages for high-resolution observation with its capability of seeing inside a sample. After developing an aberration corrector, atomic arrangements became observable using a wide range of accelerating voltages. However, the resolutions in magnetic field observations with lower accelerating voltages are not enough to observe on an atomic scale. For observing electromagnetic fields on an atomic scale using electron holography, we developed a 1.2-MV TEM equipped with a cold field-emission gun and a Corrected Electron Optical Systems (CEOS) hexapole spherical aberration corrector. Spatial resolution reached $0.043 \mathrm{~nm}$ at high-resolutions, when the sample was placed in a high magnetic field of the objective lens [1]. Under observation conditions, in which the sample was placed in a field-free position for observing a magnetic field, the spatial resolution reached $0.24 \mathrm{~nm}$ [2]. Although the spatial resolution of TEM reached a subnanometer level, magnetic field observations at these resolutions have not yet been achieved because there were additional difficulties: improving magnetic phase sensitivity and separating the electrostatic and magnetic phases at high spatial resolutions. We showed that the highest spatial resolutions for in-plane magnetic field observations was achievable by developing a pulse magnetization system for use with the aberration-corrected 1.2-MV holography electron microscope.

The phase resolution of electron holography reached $2 \pi / 1000 \mathrm{rad}$ in a vacuum area by suppressing statistical noise [3]. However, the phase resolution of the magnetic phase measured in the sample area was around $2 \pi / 200 \mathrm{rad}$ [4] because of difficulties in data acquisition from the separation of the electrostatic and magnetic phases at high resolutions. To overcome this, a pulse magnetization system was developed to reverse the magnetization in the sample without changing the geometrical configuration of the sample holder or stage referring to the electron beam. This resulted in sample drifts significantly smaller than those occurring with other separation methods.

To observe a subnanometer-resolution magnetic field, a multilayer consisting of $\mathrm{Ta}(5.0 \mathrm{~nm}) / \mathrm{CoFeB}(0.5$ $\mathrm{nm}) / \mathrm{Ta}(3.0 \mathrm{~nm}) / \mathrm{CoFeB}(1.0 \mathrm{~nm}) / \mathrm{Ta}(3.0 \mathrm{~nm}) / \mathrm{CoFeB}(2.0 \mathrm{~nm}) / \mathrm{Ta}(6.0 \mathrm{~nm})$ was prepared by sputtering deposition. TEM observation revealed that the multilayer was intermixing at the $\mathrm{CoFeB} / \mathrm{Ta}$ interfaces. The structures of these $\mathrm{CoFeB}$ and Ta layers were mainly amorphous and the Ta layer included some nanocrystalline Ta. A thin sample (45 nm thick) was observed by using electron holography. A pulse magnetic field of $207 \mathrm{kA} / \mathrm{m}$ was used to reverse the sample magnetization. The hologram fringe spacing was set to $0.22 \mathrm{~nm}$, and the reconstruction aperture was set to enable spatial information greater than $0.66 \mathrm{~nm}$ to pass through. The reconstructed phase images were aligned, and the average phases were decomposed into the electrostatic and magnetic phases.

Figures 1a and 1b show the in-plane magnetic flux distributions displayed by a cosine of the magnetic phase amplified 600 times with smoothing over the length scale of $1.43 \mathrm{~nm}$, parallel to the $\mathrm{CoFeB}$ layer. Figure 1c shows the magnetic field of an $x$-directional component. A spatial frequency perpendicular to the magnetic layer of $1 / 0.67 \mathrm{~nm}^{-1}$ was confirmed in the first Fourier transform (FFT) pattern. 
Since emergent phenomena in ferromagnetic/heavy-metal systems, such as the giant spin-Hall effect [5] and Dzyaloshinskii-Moriya interaction [6], are hot topics in spintronics, an investigation of the robustness of ferromagnetism in the $\mathrm{CoFeB}$ layers and $\mathrm{CoFeB} / \mathrm{Ta}$ interface were important. The successful separation of the electrostatic and magnetic phases provided analysis of the magnetic field as a function of the composition ratio of $\mathrm{CoFeB}$ to Ta. The results indicated that the magnetic field was not only affected by the mixing state, but also by a maximum composition ratio of $\mathrm{CoFeB}$ to $\mathrm{Ta}$ in the individual layer [7].

We developed a technique for exploring magnetic characteristics with high resolution that can be used for various kinds of fundamental research and practical applications to industrial devices.

\section{References:}

[1] T. Akashi et al., Appl. Phys. Lett. 106 (2015), p. 074101.

[2] T. Tanigaki et al., Adv. Imaging Electron Phys. 198 (2016), p. 69.

[3] T. Suzuki et al., Ultramicroscopy 118 (2012) p. 21.

[4] Y. Murakami et al., Nat. Commun. 5 (2014) p. 4133.

[5] L. Liu et al., Science 336 (2012) p. 555.

[6] S. Emori et al., Nat. Mater. 12 (2013) p. 611.

[7] T. Tanigaki et al., Sci. Rep. 7 (2017), p. 16598.

[8] Development of the 1.2MV holography microscope was supported by a grant from the Japan Society for the Promotion of Science (JSPS) through the "Funding Program for World-Leading Innovative R\&D on Science and Technology (FIRST Program)" initiated by the Council for Science, Technology, and Innovation (CSTI). Part of this research was supported by CREST, JST.

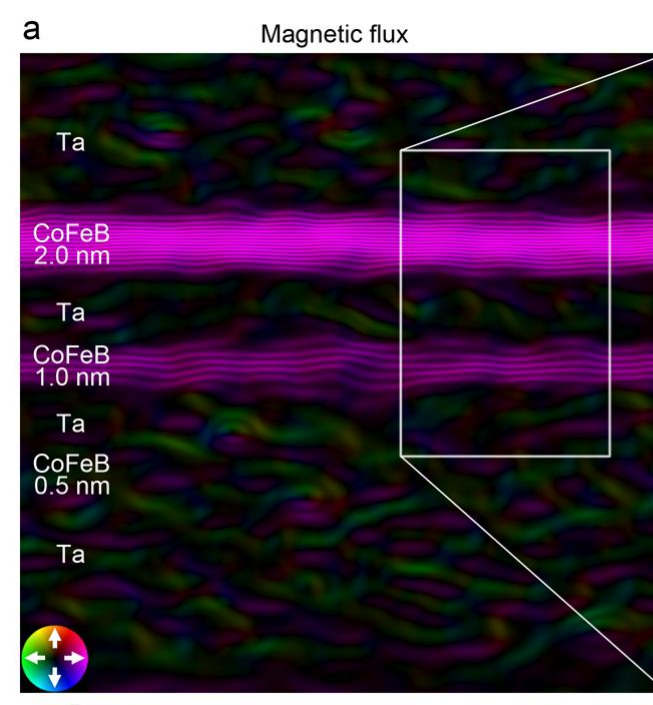

$5 \mathrm{~nm}$

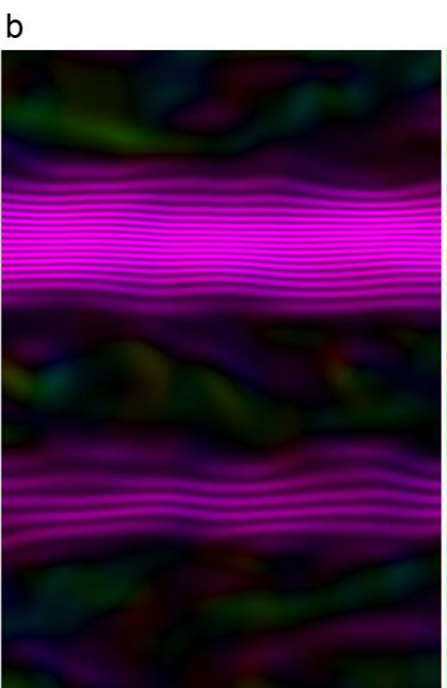

$2 \mathrm{~nm}$
C Magnetic field $B_{x}$
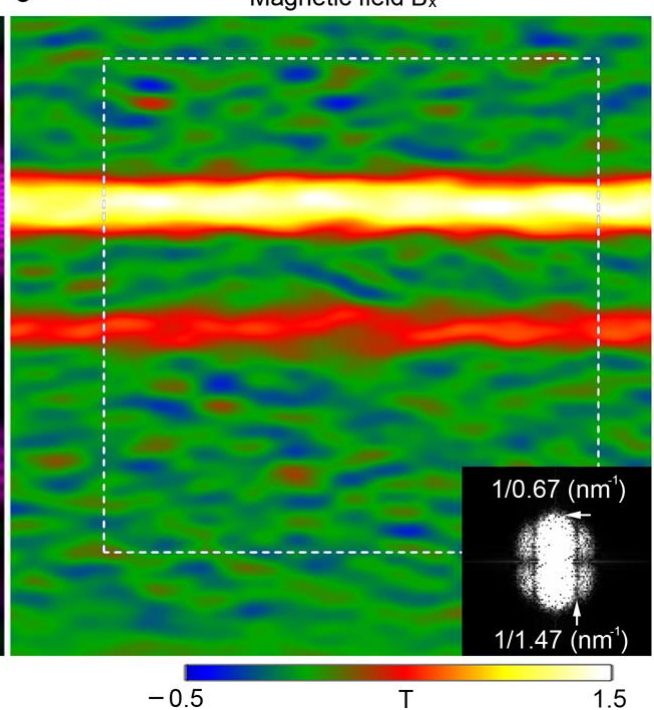

Figure 1. (a) Magnetic flux displayed by cosine of phase amplified 600 times; (b) Enlarged image of area indicated by white rectangle in (a); (c) Magnetic field of $x$-directional component. 